九州大学学術情報リポジトリ

Kyushu University Institutional Repository

\title{
Ueber Den Mechanismus Der Virusentstehung In Der Zelle
}

Yamafuji, Kazuo

Agrikulturchemischen Institut der Kyushu-Universität

Yuki, Teigen

https://doi.org/10.5109/22636

出版情報：九州大学大学院農学研究院紀要. 9 (4)，pp.317-323，1950-11. Kyushu University バージョン：

権利関係 : 
Journal of the Faculty of Agriculture, Kyūshū University, Vol 9, No. 4

November 30,1950

\section{UEBER DEN MECHANISMUS DER VIRUSENTSTEHUNG IN DER ZELLE}

Kazuo Yamafuji und Teigen Yuki

Für die Untersuchungen der chemischen Vorgänge in der lebenden Zelle haben wir seit über zehn Jahren häufig die Seidenraupe als Material verwendet. Dieses Insekt ist für die diesbezüglichen Erforschungen besonders geeignet, denn in seinem Körper erfolgen wegen des starken Wachstums im Larvenstadium und der Metamorphose in den darauffolgenden Entwickelungsperioden sehr kräftige Stoffwechselprozesse. Ferner können wir unter der Verwendung des genannten Versuchstieres einheitliche Daten erhalten, da wir eine grosse Anzahl der Raupen gleichzeitig zu züchten und also die individuellen Fehler zu vermeiden vermögen. Im Verlaufe der so wiederholten Züchtungen des Seidenspinners wurde bisweilen beobachtet, dass bei diesem Insekt eine Viruskrankheit ohne direkte Ansteckung entsteht. Nach langen eingehenderen Beobachtungen derartiger Erscheinung kamen wir zu der Annahme, dass für die Viruserkrankung nicht immer eine Infektion des Virus notwendig ist, dass nämlich eine Virusbildung auch durch irgendwelche abnorme Stoffwechselvorgänge herbeigeführt werden kann. Die Tatsachen, dass alle bisher rein hergestellten pflanzlichen sowie tierischen Viren als hochmolekulare Nucleoproteine charakterisiert worden sind, dass für die Vermehrung der Viren auf jeden Fall das Mitvorhandensein der lebenden Zellen unerlässlich ist und ferner, dass eine plötzliche weitreichende Entstehung der Viruskrankheiten oft in der Natur beobachtet wird, scheinen die oben erwähnte Annahme zu stützen. Wenn dies der Fall ist, so müssen solche Stoffwechselverschiebungen, die zur Bildung der Virusmoleküle führen, Prozesse sein, die im allgemeinen in der Gewebezelle verhältnismässig leicht geschehen können. Auf Grund der oben beschriebenen Tatsachen vermuteten 
wir weiter, dass bei der Neubildung des Virus vor allem eine Störung des Eiweissstoffwechsels und des Atmungsvorganges zu bewirken sind. Andererseits haben wir in einer ganzen Reihe von Arbeiten festgestellt, dass bei der Sauerstoffatmung in den meisten Fällen Wasserstoffperoxyd als Endprodukt gebildet wird. Nach unserer Theorie wird nämlich der terminale Prozess der Atmung folgendermassen formulert (1).

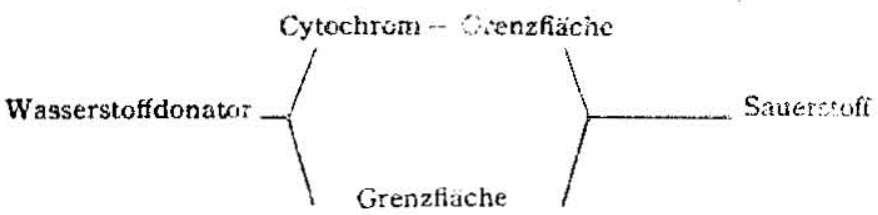

Es ist demnach anzunehmen, dass das Wasserstoffperoxyd, welches durch Verschiebung des Gleichgewichtes zwischen dem Sauerstoffverbrauch und der Katalasewirksamkeit im Gewebe überschüssig entstand, auf das normale Protoplasmaeiweiss denaturierend und ferner polymerisierend wirkt. Das so polymerisierte Nucleoprotein könnte unter den günstigsten Bedingungen die Virusnatur bekommen. Unter dieser Voraussetzung haben wir mit verschiedenen Materialien von pflanzlicher bzw. tierischer Herkunft die Wirkung der Viren auf die Atmung oder Katalase in den Zellen $(2,3)$, die Beziehung zwischen der katalatischen Aktivität und der Sauerstoffabsorption bei viruskranken Organismen (4), die Bildung des Riesenmoleküls des Eiweisses oder des Virusproteins in den lebendigen Geweben durch künstliche Mittel ( 5 bis 9 ) und die vorbeugende Fähigkeit der Katalase gegen Viruskrankheit (10) untersucht. Unsere Hypothese der spontanen Erzeugung der Virosen wurde aber durch die Versuche am Seidenspinner am deutlichst bestätigt. Wie oben erwähnt, muss bei der Entstehung des Virus in erster Linie eine Denaturierung des Nucleoproteins im Protoplasma stattfinden, ohne dass dabei das Leben der betreffenden Zelle geschädigt wird. Tatsächlich sind wir imstande, bei der Seidenraupe durch milde Erwärmung des Larvenkörpers oder durch Fütterung mit verdünnter Formaldehydlösung eine Viruserkrankung hervorzurufen. Bei der Erforschung des chemischen Mechanismus dieser Erscheinungen wurde festgestellt, dass durch solche Mittel, wie erwartet, eine Anhäufung des Wasserstoffperoxydes bewirkt wird (5). Die Ansicht, dass in 
den Bildungsvorgängen der Virusmoleküle das im Gewebe unzerstört gebliebene Hydroperoxyd eine wesentliche Rolle spielt, würde dann am klarsten erwiesen, wenn es uns gelungen wäre, durçh Behandlung der Lebewesen mit einer Hydroperoxydlösung un mittelbar eine Virusentstehdng hervorzubringen. Es ist jedoch schwer, wegen der leichten Zersetzbarkeit des Wasserstoffperoxyds, das angewandte Peroxyd auf den Eiweisskörper in der Zelle genügend stark einwirken zu lassen. In der Tat konnten wir bei der Finwirkung der Hydroperoxydlösung auf lebendige Organismen keine Neubildung des Virusproteins, wohl aber eine Bildung des höhermolekularen Eiweissstoffes nachweisen (6). Wir haben daher versucht, eine momentane Anhäufung von Wasserstoffperoxyd in der Gewebezelle dadurch zu bewirken, dass wir ein spezifisches Mittel anwandten, das auf die Katalase eine hemmende Wirkung, jedoch auf die Atmung fast keinen Einfluss ausübt. Dieser Versuch wurde unter Anwendung von Hydroxylamin mit Erfolg ausgeführt, das heisst, durch Versorgung mit einer Hydroxylaminhydrochloridlösung vermochten wir bei der Seidenlarve eine Viruskrankheit entstehen $\mathrm{zu}$ lassen (7). Aus den Ergebnissen der weiteren ausführlicheren Untersuchungen der experimentellen Produktion des Virusproteins wurde der Schluss gezogen, dass die Virusbildung in der lebendigen Zelle nach folgendem Schema verläuft (8).

Normales Nucleoprotein-Denaturiertes Nucleoprotein- Polymerisiertes Nucleoprotein ….... Virusmolekül.

Das im Vergleich mit der Katalaseaktivität überflüssig entstandene Wasserstoffperoxyd wirkt also bei diesen Vorgängen hauptsächlich als Denaturierungs- sowie Polymerisationskatalysator. Wirklich wird die Katalase in der Zelle durch Einwirkung der Viren gehemmt $(2,3)$. Dementsprechend ist also möglich, dass die Viruskrankheit durch Einnehmen von Katalase einigermassen verhütet werden kann. Auch diese Möglichkeit wurde durch die Versuche an der Seidenraupe bestätigt (9).

Obwohl unsere Theorie über den Chemismus der Bildung bzw. der Vermchrung des Virusmoleküls in der eben berichteten Weise als sicher aufgestellt werden kann, erscheint es uns doch zweckmässiger, weiterhin nachzuweisen, dass direkt unter der Einwirkung der Peroxydverbindungen auf den lebendigen Eiweisskörper 
cine Viruserkrankung herbeigeführt wird. Da bei den oben angegebenen Versuchen mit der Hydroperoxydlösung keine befriedigenden Resultate gewonnen wurden, wurde in der vorliegenden Arbeit eine solche Substanz gewählt, die im Gewebe allmählich zerlegt wird und dabei das Wasserstoffperoxyd abgibt. Wir haben nunmehr nämlich den Seidenwürmern eine geringe Menge Bariumperoxyd mit den Maulbeerblättern zusammen gegeben. Nach bestimmten Tagen zeigte sich erwarteterweise eine Anzahl viruserkrankter Individuen. Zugleich wurde hierbei gefunden, dass durch die Fütterung mit dem Bariumperoxyd nicht nur eine Erniedrigung der Katalasewirksamkeit, sondern auch eine Erhöhung der Atmungsintensität im Körper hervorgebracht wird. Infolgedessen grschieht in dem mit dem genannten Peroxyd gefütterten Larvenkörper eine überschüssige Hydroperoxydbildung sowohl bei der Peroxydzerlegung als auch beim anormalen Atmungsvorgang. Es ist alsdann nötig zu prüfen, ob das Bariumion beim Phänomen dè Virusbildung irgendeine Rolle spielt oder nicht. Deshalb haben wir die Seidenraupe mit Bariumoxyd oder Bariumchlorid versorgt. Die Versuche crgaben, dass das Chlorid eine Fähigkeit besitzt, die Larve in einen Virus-Krankheitszustand zu versetzen, während die Viruserkrankung derselben duich das Oxyd nicht beeinflusst wird. Obschon das virusbildende Vermögen des Bariumchlorides ziemlich schwach ist, ist ferner die Wirkung der Chloridverbindungen auf die Virusentstehung zu untersuchen. Es wurde deswege: schliesslich die Raupe mit Natriumchlorid bzw. Calciumchlorid gefüttert. Dabei stellte sich heraus, dass diese Verbindungen keine Krankheit hervorruft.

Durch die Versuche mit Bariumperoxyd konnte unsere Hydroperoxydtheorie der Virusbildung eine weitere wesentliche Stütze erhalten. Es wird jedoch angenommen, dass eine ausserordentlich grosse Anzahl Virosenarten im Pflanzen- und Tierreich nicht immer durch ganz gleiches Agens bewirkt $z u$ werden brauchen. Ausser den Faktoren, die im Organismus eine Wasserstoffperoxydanhäufung und sodann eine Virusbildung verursachen, wirken auch einige andere, welche ebenfalls Viren erzeugen lassen, ohne dass sie eine übermässige Hydroperoxydentstehung herbeiführen. Dergleichen Mittel müssen eine Fähigkeit haben, entweder selbst auf die Eiweissmoleküle im Protoplasma als Denaturierungs- und Aggregationskatalysator zu wirken, oder als Katalysatoren zu 
wirkende Substanzen, abgesehen von Hydroperoxyd, zu bilden. Ein Beispiel dafür bietet Bariumchlorid, obgleich es nur ein geringes diesbezügliches Vermögen besitzt. Wenn man denkt, dass die Wasserstoffperoxydbildung eine allgemeine biologische Erscheinung ist, so muss man sich natürlich vorstellen, dass eine Virusentstehung, an der das Wasserstoffperoxyd nicht beteiligt ist, ein Ausnahmephänomen darstellt. Auf jeden Fall ist es sehr bemerkenswert, dass wir einwandfrei erweisen konnten, dass die Viruserkrankung, welche im allgemeinen nur als Folge der Kontagion betrachtet worden ist, auch durch ungewöhnliche Stoffwech selvorgänge ohne Virusinfektion hervorgebracht wird.

I.

Zur Erhaltung noch kräftigerer Beweismaterialien für unsere Theorie der Neubildung des Virusproteins haben wir in der vorliegenden Untersuchung versucht, durch Einwirkung der Peroxyde selbst eine Viruskrankheit produzieren zu lassen. Obwohl es bekannt ist, dass das Wasserstoffperoxyd oder die anderen Peroxydverbiudungen auf einige organische Verbindungen, wie zum Beispiel Aldehyde, polymerisierend wirken, waren die Versuche zur experimentellen Erzeugung der eigentlichen Virosen mit der Hydroperoxydlösung erfolglos. Danach haben wir Metallperoxyde verwendet, welche in der wässerigen Iösung allmählich das Wasserstoffperoxyd entwickelten. Die Peroxyde der Alkalimetalle sind für diesen $Z$ weck ungeeignet, denn sie werden zu heftig zersetzt. Nach einer Reihe von Vorversuchen wurde Bariumperoxyd gewählt und zwar die Untersuchung zunächst am Seidenwurm ausgeführt, weil unsere Erfahrungen lehrten, dass unter den von uns bisher benutzten Pflanzen und Tieren dieses Insekt am leichtesten an einer künstlichen Viruskrankheit erkrankt. Die Larven der Rassen (Japan $115 \times$ Japan 12) $\times$ China 108 wurden nach der zweiten Häutung in mehrere Gruppen geteilt und mit Bariumperoxydlösungen von verschiedenen Konzentrationen benetzten Maulbeerblätter gegeben. Hin und wieder wurden die Atemgrösse sowie Katalasewirksamkeit der Raupen bestimmt (Tabelle I).

Bei einer Bariumperoxydkonzentration von mehr als $\mathrm{m} / 5$ wurde die Lebenstätigkeit der Larve ziemlich beeinträchtigt. Da die Wasserstoffionenkonzentration der Körperflüssigkeit des Seiden- 
spinners bei etwa pH 6,5 liegt (11), kann das in den Raupenkörper aufgenommene Bariumperoxyd unter Abgabe des Wasserstoffperoxydes gespaltet werden. Der unerwartet hohe Prozentsatz der durch verwandtes Bariumsalz erkrankten Raupen wird dadurch erklärt, dass das infolge der durch die Peroxydfütterung bewirkten Atmungsstörung gebildete Wasserstoffperoxyd zusammen mit dem auf eben beschriebene Weise entstandenen auf das Protoplasmaeiweiss einwirkt.

Tabelle I.

\begin{tabular}{|c|c|c|c|}
\hline Gefüttert mit : & $\mathrm{m} / 10 \mathrm{BaO}_{2}$ & $\mathrm{~m} / 20 \quad \mathrm{BaO}_{2}$ & Wasser \\
\hline Relative Atmungsintensitiat ................. & 141 & - & 100 \\
\hline Relative Katalaseaktivität ... & 61 & 一 & 100 \\
\hline Zahl der viruserkrankten Iarven in $\%$ & 70 & 5.5 & 0 \\
\hline
\end{tabular}

II.

Es besteht die Möglichkeit, dass die in den obenerwähnten Versuchen beobachtete viruserzeugende Fähigkeit des Bariumperoxydes nicht auf der Wirkung des von diesem Salz herrührenden Hydroperoxydes, sondern auf derjenigen der Metallionen des Bariums beruht. Deswegen wurden in dieser Versuchsreihe die Larven mit Maulbeerblättern versorgt, die vorher mit Bariumsalzlösungeiz befeuchtet worden waren (Tabelle II).

Tabelle II.

\begin{tabular}{|c|c|c|c|c|}
\hline Gefüttert mit: & $\mathrm{m} / \mathrm{s} \mathrm{Ba}$ & $8 \mathrm{BaCl}_{2}$ & $\mathrm{~m}_{/} / \mathrm{B} \mathrm{BaO}$ & Wasser \\
\hline Relative Atmungsintemsitä ............... & 104 & 108 & 121 & 100 \\
\hline Relative Katalaseaktivitat $\ldots \ldots \ldots \ldots \ldots$ & 97 & 105 & 74 & 100 \\
\hline Zahl der viruserkrankten Larven in $\%$ & 0 & 11 & 52 & 0 \\
\hline
\end{tabular}

Durch die Einwirkung des Bariumchlorides von der angewandten Konzentration starben eine kleine Anzahl Raupen an einer Krankheit, welche nicht virusartig ist. Ausserdem wurden durch das Chlorid die Atmung sowie Katalase etwas erhöht. Aus den hier erhaltenen Befunden lässt sich schliessen, dass das Bariummetall selbst kein virusproduzierendes Vermögen hat. 
III.

Im Anschluss an die Experimente mit dem Bariumchlorid haben wir schliesslich untersucht, ob durch das Chlorion eine Viruserkrankung bewirkt wird, indem wir die Seidenlarven mit Maulbeerblättern fütterten, die mit den Lösungen des Natrium-, Calcium- oder Bariumchlorides benetzt worden waren (Tabelle III).

Tabelle III.

\begin{tabular}{|c|c|c|c|c|}
\hline Gefüttert mit: & $\mathrm{m} / 15 \mathrm{~N}$ & $5 \mathrm{Ca}$ & $5 \mathrm{BaCl}_{\mathrm{a}}$ & Wasser \\
\hline Zahl der viruserkrankten Larven in $\%$ & 0 & 0 & 7 & 0 \\
\hline
\end{tabular}

Aus diesen Versuchen geht hervor, dass die oben beschriebene Aktivität des Bariumchlorides nicht auf die Wirkung des Halogenions zurückzuführen ist. Die in den vorliegenden Untersuchungsreihen mit den Bariumsalzen festgestellte Viruskrankheit war auf alle Fälle mit der in den bisherigen Arbeiten stets beobachteten Polyederviruskrankheit identisch. Wir konnten also mit Hilfe verschiedenartiger Mittel, unserer Theorie entsprechend, immer dieselbe Viruserkrankung herbeiführen.

Die Arbeit wurde durch ein Stipendium des Unterrichtsministeriums unterstützt.

(Aus dem Agrikulturchemischen Institut der Kyushu-Universität in Fukuoka, Japan)

\section{LITERATUR}

1) K. Yamafuji u. T. Fujiki, Biochem. Zs. 317, 99, 1944.-2) K. Yamajuji, K. So u. K. Soo, ebenda 311, 203, 1941.-_-3) K. Yamafuji, K. So u. T. Kitano, ebenda 315, 415, 1943.--4) K. Yamafuji, K. So u. K. Nagano, ebenda 315, 411, 19.43...-5) $K$. Yamafuji u. Y. Kosa, ebenda 317, 81, 1944,--6) K. Yamafuji u. F. Yoshihara, ebenda 317, 87, 1944.—7) K. Yamafuji t. Y. Shirozu, ebenda 317, 94. 1944-——8) K. Yama. fuji u. T. Cho, ebenda 318, 95, 1947._-9) K. Yamafuji u. T. Fujiki, ebenda 318, 101, 1947. — 10) K. Yamafuji u. T. Yuki, ebenda 318. 107, 1947. - 11) K. Yamafuji, Bul1. agricult. chem. Soc. Japan 9, 19, 1933. 